\title{
Smooth Transition-Based Control of Encounter-Type Haptic Devices
}

\author{
Franck Gonzalez $^{1234}$, Wael Bachta ${ }^{234}$ and Florian Gosselin ${ }^{1}$
}

\begin{abstract}
Encounter-type devices have been introduced to solve one of the main drawbacks of common haptic interfaces i.e. their limited transparency in free space and their restricted ability to render realistic transitions between free space and contact. Advantageously, encounter-type interfaces collide with fingers and display interaction forces only if a contact occurs in the virtual environment. In free space, their end-effectors are mechanically detached from the operator and closely track his fingers, allowing for a perfect transparency. This paper introduces a control scheme that handles the transition between tracking and force display modes in a smooth way. Experimental results using a custom encounter-type interface are also given. The obtained results show the efficiency of the proposed control law when compared to the conventional switch-based control usually found in the literature.
\end{abstract}

\section{INTRODUCTION}

Haptic interfaces are purposed for natural interactions between an operator and a virtual or a remote environment [1], [2], [3], [4], [5]. They should enable the user to sense correctly even high impedances of distant or virtual objects, and also display negligible impedance when there is no contact with the environment [6]. The latter requirement calls for an interface with low inertia links and motors, along with small gear ratios. These specifications may be in conflict with rigidity and force level requirements. Force control strategies can also be implemented to reduce the apparent impedance in free space motion [7]. Their performances are however limited.

In this context, Encounter-Type Haptic Displays (ETHDs) are of interest as their working principle relies on a mechanical detachment between the user and the interface in free space motion [8], [9], [10], making them perfectly transparent. As shown in Fig. 1, the interface closely tracks an operator's finger thanks to a thimble-like end-effector featuring embedded optical distance sensors and encounters it only when a contact occurs in the virtual or remote environment [11].

\footnotetext{
${ }^{1}$ CEA, LIST, Interactive Robotics Laboratory, F-91190 Gif-sur-Yvette, FRANCE.

2 Sorbonne Universités, UPMC Univ Paris 06, Institut des Systèmes Intelligents et de Robotique, F-75005, Paris, FRANCE.

${ }^{3}$ CNRS, UMR 7222, Institut des Systèmes Intelligents et de Robotique, F-75005, Paris, FRANCE.

${ }^{4}$ INSERM, UI 1150, Institut des Systèmes Intelligents et de Robotique, Equipe Agathe, F-75005, Paris, FRANCE.

\{franck.gonzalez, florian.gosselin\}ecea.fr

wael.bachta@isir.upmc.fr

This research was partly supported by the "Agence Nationale de la Recherche" (Mandarin project - ANR-12-CORD-0011, labeled by "Cap Digital Paris Région", the French cluster for digital contents and services), and partly accomplished within the laboratory of excellence SMART supported by French state funds managed by the ANR within the Investissements d'Avenir program (ANR-11-IDEX-0004-02).
}

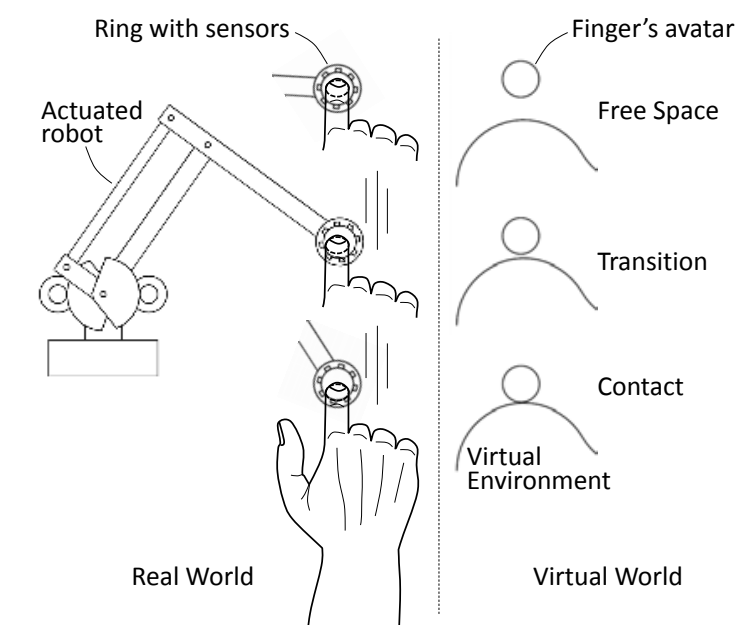

Fig. 1. Main components and operating principle of close-tracking ETHDs

Various technological solutions can be used for the design of this thimble. To measure the motion of the operator's fingertips, each of the end-effectors of the Encounter-Type hand exoskeletons for teleoperation in [12], [13] is equipped with an optical sensor that records the position of a thin reflecting plate pushed against the nail by means of a spring. The thin plate and the spring are chosen so that the force exerted on the finger during free space motion be negligible. In [11], [14], optical-fiber sensors matrices are used to estimate the finger position in a $2 \mathrm{D}$ ring and a $3 \mathrm{D}$ cap mounted on simple serial ETHDs.

In the literature, a position control scheme is usually adopted to track the movements of the user when no contact is required and a force display control is used to render the environment after contact. A simple switch handles the transition between the two control modes. In this paper, the limits of this conventional approach are discussed before proposing a smooth transition-based control law. The importance of the transition is highlighted since it has a key role in the naturalness of the haptic rendering.

The remainder of the paper is organized as follows: Section II presents the switch-based control law as implemented in the literature, then discusses its drawbacks and describes a smooth transition-based control scheme intended to overcome its shortcomings. Then Section III gives an experimental assessment of the two control laws. A 2-DoF ETHD used as an experimental set-up is presented, followed by the results of the experiments. Finally, Section IV concludes and discusses future work. 


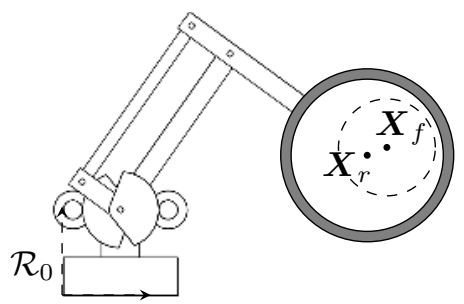

Fig. 2. Schematic 2D ETHD with reference frame and points. Grey circle: ring. Dashed circle: finger.

\section{Control Laws}

In this section, the conventional switch-based control law is first presented and its drawbacks are pointed out. Then a smooth transition-based control law is presented, which cuts off with the raised shortcomings.

\section{A. Switch-Based Control Law}

The conventional ETHD control law is composed of a position tracking mode in free space and a force display for the rendering of the environment. This subsection describes these two control modes as well as the switch modality.

1) Finger Tracking in Free Space: Let $\boldsymbol{X}_{r / 0}$ and $\boldsymbol{X}_{f / 0}$ denote the centers coordinates of respectively the robot endeffector and the finger. Both are expressed in a frame attached to the robot base (see Fig. 2). In this control mode, the goal is to maintain the finger in the center of the ring. The tracking error $\epsilon_{X}$ (see Fig. 3) is therefore directly obtained by measuring $\boldsymbol{X}_{r / f}$, which is the position of the ring center with respect to the finger.

Expressing the error in the joints space allows for an easier tuning of the controller since the interface dynamics are less coupled in this representation [15]. In case of a close tracking, $\epsilon_{X}$ is small so it can be expressed in the articular space as follows:

$$
\boldsymbol{\epsilon}_{\theta}=\mathbf{J}^{-1}(\boldsymbol{q}) \cdot \boldsymbol{\epsilon}_{X}
$$

with $\mathbf{J}(\boldsymbol{q})$ the Jacobian matrix of the robot in the reference frame.
This error is sent to a Proportional-Derivate controller which provides the robot with the reference torque $\tau_{t}$ :

$$
\boldsymbol{\tau}_{t}=\left(\boldsymbol{K}_{t}+\boldsymbol{B}_{t} s\right) \boldsymbol{\epsilon}_{\theta}
$$

Finally, its joints positions are used to compute the position of the ring's center $\boldsymbol{X}_{r / 0}$ through a geometric model.

2) Transition Between Modes: As shown in Fig. 3, the switching between tracking mode (1)-(2) and contact mode (6)-(7) (see below) is triggered by $\boldsymbol{F}_{e}$ through the matrix $\mathbf{S}$, defined as follows:

$$
\mathbf{S}=\left[\begin{array}{cc}
e^{-\alpha\left\|\boldsymbol{F}_{e}\right\|} & 0 \\
0 & e^{-\alpha\left\|\boldsymbol{F}_{e}\right\|}
\end{array}\right]
$$

with $\alpha$ a dimensionless number so that $\forall \boldsymbol{F}_{e}, \alpha \gg\left\|\boldsymbol{F}_{e}\right\|$. This way, when the finger's avatar encounters a virtual object, $\left\|\boldsymbol{F}_{e}\right\| \neq 0$. The switch is triggered: the robot is no more influenced by the tracking $\left(e^{-\alpha\left\|\boldsymbol{F}_{e}\right\|} \simeq 0\right.$ ) but only by contact forces. When the finger's avatar leaves the virtual object, $\left\|\boldsymbol{F}_{e}\right\|=0$, the force display is set off and the tracking torque reference is sent to the servo-drive with a unit gain.

Several works have been dedicated to the computation of distances in a virtual environment [16], [17]. Let $d_{f / e}$ be the distance of the finger's avatar periphery to the closest point of a virtual object, along the normal $\boldsymbol{n}$ to the virtual object at this point (computed from the position of the finger's center $\boldsymbol{X}_{f / 0}$ and $R_{f}$ ). We define a compensatory tracking term $\boldsymbol{\Delta} \boldsymbol{X}=f\left(d_{f / e}\right)$ by (see Fig. 4$)$ :

$$
\begin{cases}\text { if } d_{f / e}>R_{r}-R_{f} & \boldsymbol{\Delta} \boldsymbol{X}=\mathbf{0} \\ \text { if } d_{f / e} \in\left[0 ; R_{r}-R_{f}\right] & \boldsymbol{\Delta} \boldsymbol{X}=\left(R_{r}-R_{f}-d_{f / e}\right) \boldsymbol{n}\end{cases}
$$

with $R_{r}$ the radius of the ring. The Cartesian error $\epsilon_{X}$ is modified as follows:

$$
\boldsymbol{\epsilon}_{X}=-\boldsymbol{X}_{r / f}-\boldsymbol{\Delta} \boldsymbol{X}
$$

This way, when $d_{f / e}>R_{r}-R_{f}$, the finger is kept centered with the ring. Then, for $0<d_{f / e} \leq R_{r}-R_{f}$, the ring's inner surface approaches the finger linearly. Finally, when the finger's avatar comes into contact with the virtual environment, the ring touches the finger.

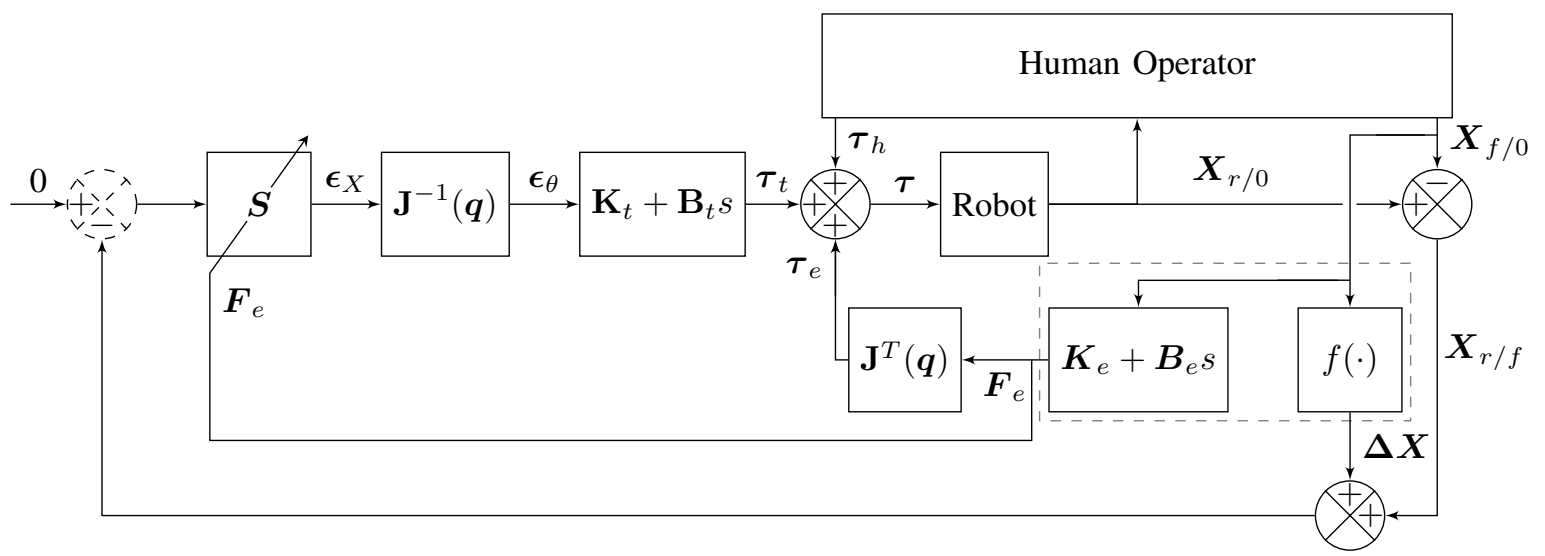

Fig. 3. Control law with switch, in the framework of [11]. S: Switch, see (3). Dashed area: Virtual Environment. $f(\cdot)$ : tracking compensation, see (4). 


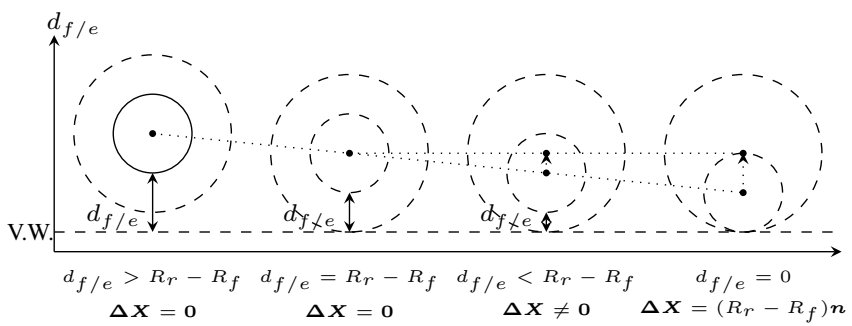

Fig. 4. Positions of the avatars of the finger and ring in function of the distance to the virtual wall. Dashed circles: ring; plain circles: finger; singlesided arrows: $\boldsymbol{\Delta} \boldsymbol{X}$. V.W.: virtual wall.

3) Force Feedback in Contact: When the user's avatar encounters a virtual object, the interface must render the associated interaction force $\boldsymbol{F}_{e}$. Assuming a viscoelastic compliant virtual environment without tangential friction, this force is defined as a unilateral spring-damping set, as follows:

$$
\begin{cases}\text { if } d_{f / e} \geq 0 & \boldsymbol{F}_{e}=\mathbf{0} \\ \text { if } d_{f / e}<0 & \boldsymbol{F}_{e}=\left(\mathbf{K}_{e}+\mathbf{B}_{e} s\right) d_{f / e} \boldsymbol{n}\end{cases}
$$

The torque $\tau$ applied on the interface joints is given by:

$$
\boldsymbol{\tau}=\boldsymbol{\tau}_{h}+\boldsymbol{\tau}_{e}
$$

where $\boldsymbol{\tau}_{h}$ is the torque due to the force $\boldsymbol{F}_{h}$ applied by the finger on the end-effector, and $\boldsymbol{\tau}_{e}$ is a torque corresponding to the interaction force $\boldsymbol{F}_{e}$. This torque is given by:

$$
\boldsymbol{\tau}_{e}=\mathbf{J}^{T}(\boldsymbol{q}) \boldsymbol{F}_{e}
$$

\section{B. Smooth Transition-Based Control Law}

In this subsection, the drawbacks of the switch-based control law are first discussed. Then, a smooth-transition based control law is proposed.

1) Limitations of Previous Approach: To achieve a quality rendering, the switching must occur exactly when the virtual or remote object is encountered. At this very moment, the interface's end-effector must be in contact with the finger of the operator. In the switch-based control law, the switch time and location are based on the knowledge of the finger radius. This assumes that the finger contour is cylindrical: this assumption may not be robust. Indeed, a finger geometry is more complex and deformable, so it is likely to change during the contact. In order to highlight this issue, two scenarios illustrating the effects of a wrong estimation of the finger radius are discussed in the sequel (Fig. 5):

- Over-estimation of the finger radius $R_{f}$. As the finger's avatar is larger than the real finger, a contact would occur in the virtual world before the operator's finger touches the ring. A force $F_{e}$ would thus be displayed by the interface although the finger is not in contact with the ring. In other words, the interface would move back and hit the operator's finger. Thus the user would feel parasitic movements of the robot bumping into his finger, modifying the behavior of the environment.
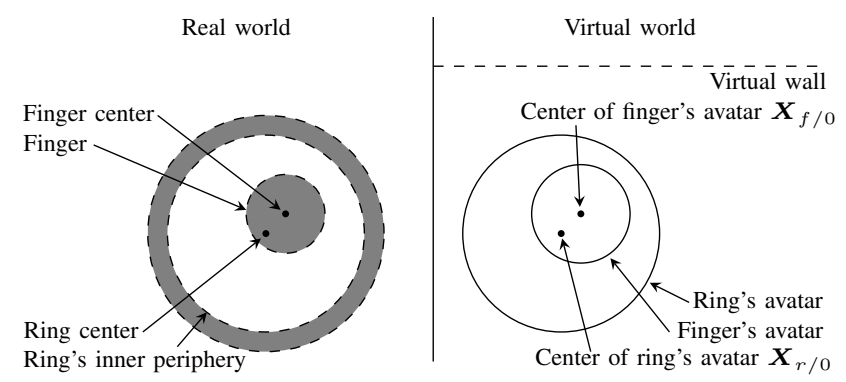

Fig. 5. Main factors influencing the tracking and force feedback in the real world, and their counterparts in the virtual environment. Note that the ring's avatar is of the same size as the real ring inner periphery, which is perfectly known, whereas the size of the finger's avatar, which is estimated, can differ from the real one.

- Under-estimation of the finger radius $R_{f}$. This would translate into the finger hitting the end-effector before its avatar come into contact with the virtual wall. In this case, the user would feel as if the virtual object were made of two layers. The first sensation would correspond to a parasitic force $\boldsymbol{F}_{p}=\left(\mathbf{K}_{t}+\mathbf{B}_{t} s\right)\left(R_{r}-\right.$ $\left.R_{f}-d_{f / e}\right) \boldsymbol{n}$ due to the tracking mode, which would not be disabled as the finger's avatar would not be in contact with the virtual wall yet. When the finger's avatar would finally encounter the virtual wall, the second (purposed) rendering would be sensed.

These behaviors decrease the transparency of the interface and limit the interaction quality. Their effects may be minimized by enhancing the end-effector's tracking performances in order to better detect the finger geometry. However, this would involve expensive solutions that cannot be considered in a majority of applications. In the sequel, a smooth transition control law, independent on the finger geometry, is proposed to overcome these limitations without any change on the hardware.

2) Proposed Solution: To achieve a smooth transition, the tracking control law component should be set off at the very instant when the finger avatar collides with the virtual environment. In the switch-based control law, this is achieved by servoing the robot end-effector center to a distance $R_{r}-R_{f}$ to the virtual environment at the instant of the collision. At the same time, the tracking control component is disabled using $\mathbf{S}$. Since it is based on the finger radius, the efficiency of this method is limited. Another way of implementing the transition consists in adapting the tracking control component in function of the distance $d_{f / e}$ between the avatar of the finger and the virtual object. When this distance is large, the full tracking control magnitude is used. Conversely, when this distance is small, only a very little amount of this component is used. The interface can no longer be centered around the finger and the latter can collide with the ring. This method is practically implemented 


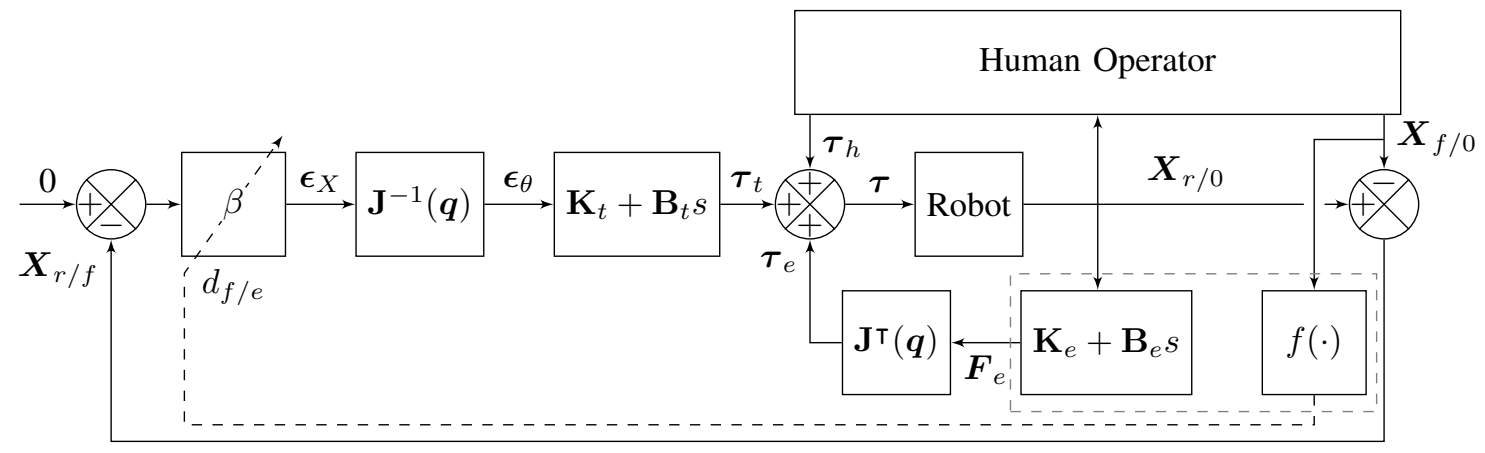

Fig. 6. Smooth control law without switch.

using an adaptive gain $\beta$ which depends on $d_{f / e}$ (see Fig. 6):

$$
\begin{cases}\text { if } d_{f / e}>R_{r} & \beta=1 \\ \text { if } d_{f / e} \in\left[R_{r}-R_{f, \max } ; R_{r}\right] & \beta=1-0.99 \frac{R_{r}-d_{f / e}}{R_{f, \max }} \\ \text { if } d_{f / e}<R_{r}-R_{f, \max } & \beta=0.01\end{cases}
$$

with $R_{f, \max }$ chosen so that $\forall R_{f}, R_{f, \max }>R_{f}$. Also, $d_{f / e}$ is computed with $R_{f, \max }$, so the tracking component is reduced progressively. Using this adaptation, the tracking force would be unchanged as long as $d_{f / e}>R_{r}$. When $R_{r}-R_{f, \max } \leq d_{f / e} \leq R_{r}$, the ring's avatar would come into contact with the virtual wall. Finally, when $0 \leq d_{f / e} \leq$ $R_{r}-R_{f, \max }$ and the operator encounters the ring, $\beta=0.01$ and the tracking effect would be almost canceled, whereas $\left\|\boldsymbol{F}_{e}\right\| \neq 0$.

We also propose that the force display depend on the ring's avatar rather than on the finger's to avoid relying on the less known finger radius, as follows:

$$
\boldsymbol{F}_{e}=\left(\mathbf{K}_{e}+\mathbf{B}_{e} s\right) d_{r / e} \boldsymbol{n}
$$

with $d_{r / e}$ the distance between the ring's avatar and the closest point of the virtual object along $\boldsymbol{n}$. This way, the ring should remain at the surface of the virtual object and wait for the finger to encounter it. The control is expected to be more stable as the ring's geometry is better known and do not undergo any deformation or non-measurable motion.

\section{EXPERIMENTAL RESULTS}

This Section describes an experimental setup on which the control schemes are implemented in turn, in order to further investigate the assets and restrictions of the proposed approaches.

\section{A. Experimental Setup}

In order to compare the different control laws, an encounter-type interface is developed. It consists in a $2 \mathrm{D}$ haptic interface equipped with a custom end-effector. The hardware as well as the software of this interface are described in this section.

1) Robot: An optimized version of a $2 \mathrm{D}$ sub-structure saved from a 6DOF parallel robot available in our laboratory is used [18]. This robot, originally intended for telesurgery, is highly transparent and its performances are in adequacy with the simulation of fine manipulation tasks in which realistic contact rendering is of primary importance, hence intermittent contact potentially interesting. As shown on Fig. 7, the robot is placed so that its workspace lies in a vertical plane. It is powered with two Maxon RE-35 DC motors equipped with 1,000 pts incremental optical encoders.

2) End-effector: The robot is equipped with a ring-shaped end-effector adapted to 2D intermittent contact (Fig. 8). Sixteen Vishay VCNL4000 infrared proximity sensors are distributed over the inner surface of the ring. They are set up behind diaphragms which limit their fields of view in order to avoid crosstalk during measures. If a finger is placed inside the ring, its center location can be computed at a rate of $300 \mathrm{~Hz}$ using the sensors' data, with a precision of $\pm 0.3 \mathrm{~mm}$ (note that for simplicity and computation time considerations, even if the end-effector can reconstruct the finger contour in theory, only the position of the finger's center is estimated here). An inner diameter of $24 \mathrm{~mm}$ is chosen, which has been shown to be sufficient to allow the device to track a finger at medium speeds (see below).

3) Control Architecture: The proximity sensors measurements are retrieved by eight ATMega328P microcontrollers which transfer the data to the haptic interface controller through a fast serial bus at a rate of $400 \mathrm{kbps}$. An estimate of the finger's location is computed as the center of the polygon obtained from the measurements. The controller is composed of a PC104 computer running Xenomai realtime operating system and a servo-drive controlling the two motors. A custom software based on the TAO telerobotics library [19] acquires the state of the interface, computes the finger's position and sends the reference torques to the servo-drive at a rate of $1 \mathrm{kHz}$. To handle the mismatch between the control loop $(1 \mathrm{kHz})$ and the acquisition $(300 \mathrm{~Hz})$ rates, a Kalman filter dedicated to extrapolating the finger's position is implemented.

\section{B. Implementation of the Switch-Based Control Law}

A first experiment of encountering a virtual wall is implemented using the switch-based control scheme. The tracking gains are set to:

$$
\mathbf{K}_{t}=\left[\begin{array}{cc}
20 & 0 \\
0 & 15
\end{array}\right] \quad \mathbf{B}_{t}=\left[\begin{array}{cc}
0.65 & 0 \\
0 & 0.3
\end{array}\right]
$$




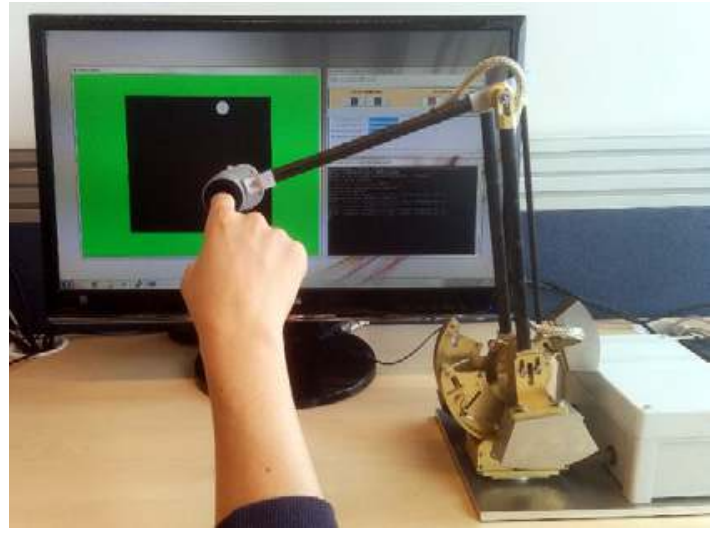

Fig. 7. Overview of the 2D ETHD used during the experiments. The 2D virtual world appears at the background: the finger's avatar is represented by a white disk, free space appears in black and the walls in green.

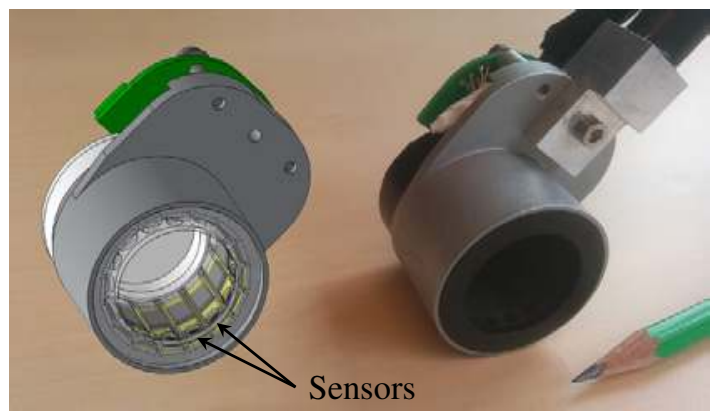

Fig. 8. CAD and closeup view of the 2D end-effector.

with $\mathbf{K}_{t}$ in $\mathrm{Nm} / \mathrm{rad}$ and $\mathbf{B}_{t}$ in Nms/rad. These gains correspond to an equivalent stiffness of 240 to $320 \mathrm{~N} / \mathrm{m}$ at the center of the robot's workspace. In practice, these gains are the maximum values guaranteeing the robot stability in free space, allowing for a finger tracking at speeds up to $0.45 \mathrm{~m} / \mathrm{s}$ and accelerations up to $10 \mathrm{~m} / \mathrm{s}^{2}$. The Cartesian gains of the environment are set up to common values allowing for the user to feel a high stiffness:

$$
\mathbf{K}_{e}=\left[\begin{array}{cc}
1,000 & 0 \\
0 & 1,000
\end{array}\right] \quad \mathbf{B}_{e}=\left[\begin{array}{cc}
10 & 0 \\
0 & 10
\end{array}\right]
$$

with $\mathbf{K}_{e}$ in $\mathrm{N} / \mathrm{m}$ and $\mathbf{B}_{e}$ in $\mathrm{Ns} / \mathrm{m}$. Note that the maximum stable gains after contact are three to four times higher than the gains in free space as in this case, the reference variable is the robot's position acquired by the actuators' encoders at $1 \mathrm{kHz}$ whereas before contact, it is the finger's position acquired by the proximeters at only $300 \mathrm{~Hz}$ and with a lower accuracy.

The result is displayed in Fig. 9. The positions and peripheries of the finger's avatar and ring are recorded along with $\boldsymbol{F}_{e}$ and $\boldsymbol{F}_{t}$ (i.e. the operational space projection of $\boldsymbol{\tau}_{t}$ ). The center and inner periphery of the ring and the virtual wall are also presented (see Fig. 5).

1) Analysis: From 0 to $35 \mathrm{~mm}$, the finger's avatar is far from the virtual wall. Therefore the ring is tracking the finger, so the ring's center and the finger's avatar center are close. During this phase, $\boldsymbol{F}_{t}$ oscillates up to $1.5 \mathrm{~N}$ due to limitations
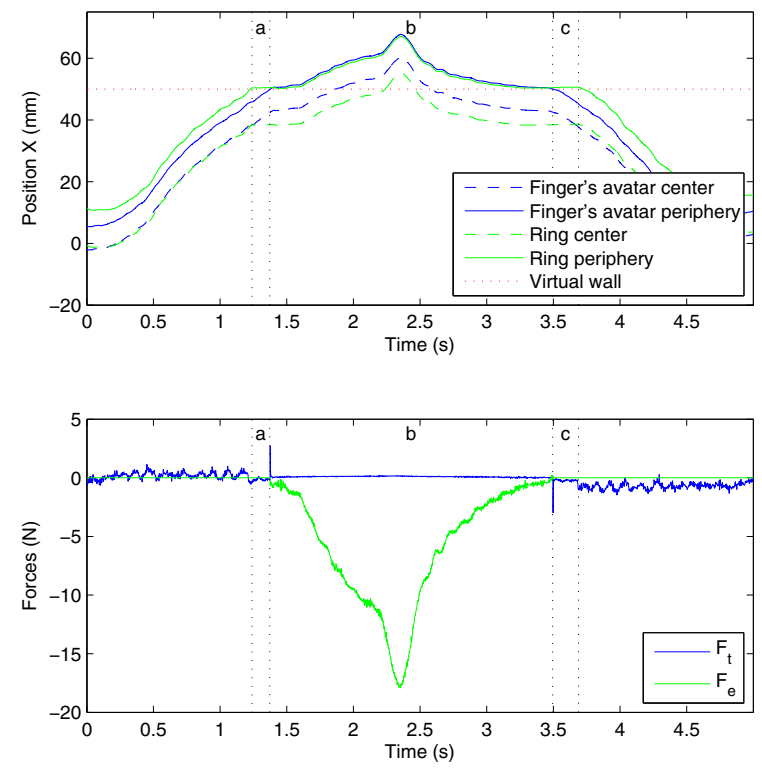

Fig. 9. 1D displacement with switching control law and finger geometry correctly estimated $\left(R_{f}=7.5 \mathrm{~mm}\right)$.

on the tracking system frequency. However this is of no influence since the ring never collides with the finger. As the finger's avatar approaches the virtual wall, $\Delta X$ increases so that the finger center no longer coincides with the center of the ring whereas their peripheries become close (see area 9a). When the finger's avatar comes into contact with the virtual wall (i.e. at $1.4 \mathrm{~s}),\left\|\boldsymbol{F}_{t}\right\| \simeq 0$ and $\left\|\boldsymbol{F}_{e}\right\| \gtrsim 0$ which means that the finger is effectively in contact with the ring (area 9b). If the operator pushes the wall, $\left\|\boldsymbol{F}_{e}\right\|$ increases and the interface behaves like a common haptic device. When leaving the virtual environment, $\left\|\boldsymbol{F}_{e}\right\|$ decreases to 0 and the tracking turns on (area 9c).

2) Effects of finger radius Estimation Errors: The above results tend to prove that when the finger geometry is properly estimated, the control law with switch works suitably. However one can note that even in this case, some force overshots of about $3 \mathrm{~N}$ can be observed (Fig. 9, end of area 9a and beginning of area $9 \mathrm{c}$ ). In practice, these behaviors alter the haptic sensation since they can be felt by the user.

Moreover, a real finger is not cylindrical and is also deformable, so its geometry vary necessarily during the contact and is not perfectly known. In this sense and following the hypotheses on the behavior of the system presented on Section II-B, two other experiments are carried out to evaluate the influence of finger radius estimation errors.

- Over-estimation of $R_{f}$ : In this experiment, $R_{f}$ is set to $11 \mathrm{~mm}$ whereas the radius of the operator's finger is of about $7.5 \mathrm{~mm}$ (the error is purposely set to a large value to emphasize the phenomenon). The results are reported in Fig. 10. As the finger's avatar is larger than the real finger, a contact occurs in the virtual world before the operator's finger touches the ring, as shown in the beginning of area 10a. A force $F_{e}$ is thus displayed by the interface although the finger is not in contact with 

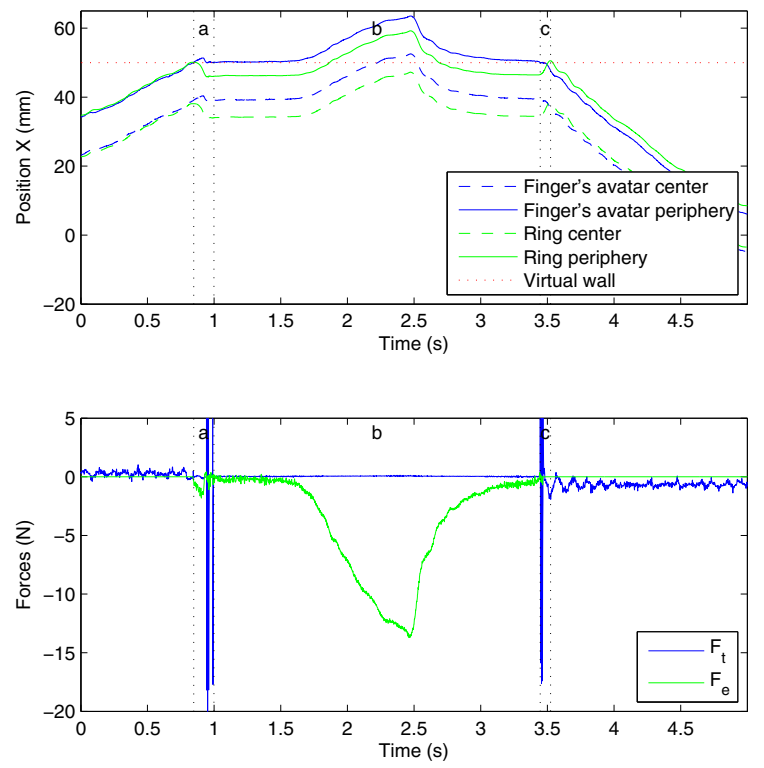

Fig. 10. 1D displacement with switching occurring too early $\left(R_{f}=\right.$ $11 \mathrm{~mm}$, over-estimated).

the ring. In other words and as previously hypothesized, the interface moves back and hits the operator's finger to take up their gap, at $1 \mathrm{~s}$. On this impulsion, the finger's avatar moves back and the tracking resumes, and so on, giving rise to the parasitic nonlinearities observed in area 10a. Then the force feedback is felt as realistic in contact (area 10b). One can note that in this phase the over-estimation of the finger radius appears as the finger periphery is several millimeters above the ring's. The same phenomenon as in area 10a arises in area $10 \mathrm{c}$ when passing back from contact to tracking, but there the transition from contact to free space is felt as realistic since the nonlinearities occur while the ring disconnects from the finger and moves back before tracking it normally.

- Under-estimation of $R_{f}$ : In this experiment, $R_{f}$ is set to $3 \mathrm{~mm}$ whereas the radius of the operator's finger is of about $7.5 \mathrm{~mm}$ (here again the error is purposely large). The results are reported in Fig. 11 and are correlated with the theoretical assumptions: as the finger's avatar is smaller, the operator's finger hits the ring before the finger's avatar comes into contact with the virtual wall, here at about $0.5 \mathrm{~s}$. Since the system did not switch yet, the operator feels a repulsive force corresponding to the tracking effort (see $F_{t}$ on area 11a). When the finger's avatar periphery finally crosses the virtual wall, at $1.1 \mathrm{~s}$, the system switches to contact mode and the second (purposed) rendering is sensed (area 11b). Thus the operator feels the virtual object as if it were made of two different layers. The same transition is observed when moving away from the virtual wall (area 11c).

The above experiments confirm that an error on the estimation of the finger radius at the contact location can lead to parasitic behaviors which can decrease the realism
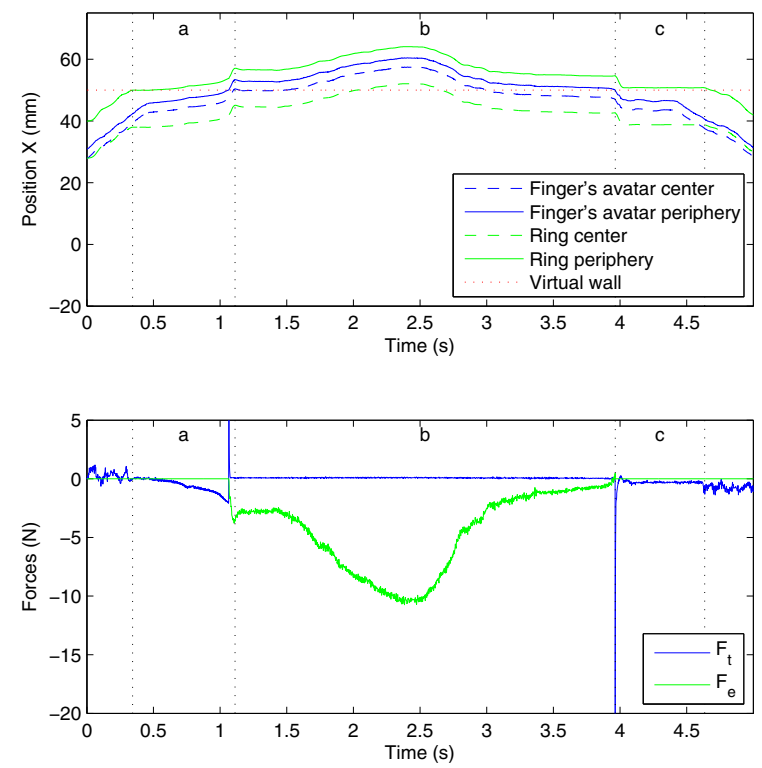

Fig. 11. 1D displacement with switching occurring too late $\left(R_{f}=3 \mathrm{~mm}\right.$, under-estimated).

of the interaction through the haptic device. These effects may be minimized by enhancing the end-effector's tracking performances in order to better detect the finger geometry. However, this would involve expensive solutions that cannot be considered in a majority of applications.

\section{Implementation of the Smooth Control Law}

The results of an experiment using the proposed smoothed control scheme are given in Fig. 12. The virtual environment is defined as before and the controller's gains are unchanged compared with the switch-based control law. According to (9) and in order for the transition to be independent from most users, we purposely set $R_{f, \max }$ to $11 \mathrm{~mm}$ (from [20], it could be argued that this value could be taken as a finger radius' upper bound).

From 0 to $35 \mathrm{~mm}$ the behavior of the system is unchanged as it is pure tracking. On area 12a the movement of the ring is stopped whereas the finger continues till it encounters the ring (the finger's avatar periphery is not displayed as it is not taken into account in the control law). The influence of $\beta$ is noticeable as it almost cancels $\boldsymbol{F}_{t}$ when the finger approaches the virtual object (area 12a). No peak of $\boldsymbol{F}_{t}$ nor $\boldsymbol{F}_{e}$ can be seen. In area $12 \mathrm{~b}, \boldsymbol{F}_{e}$ is provided according to (10) and increases more smoothly than in the previous experiments, since it is computed using $\boldsymbol{X}_{r}$. Thus the behavior of this control law appears to be better than the switch-based one.

\section{CONCLUSION}

In this paper, we presented how an existing state-ofthe-art haptic device has been used to build an encountertype interface. To this purpose, a custom ring with position measurement capabilities has been developed. A switchbased control law has been implemented in the developed setup. It consists in a position tracking mode enabled during 

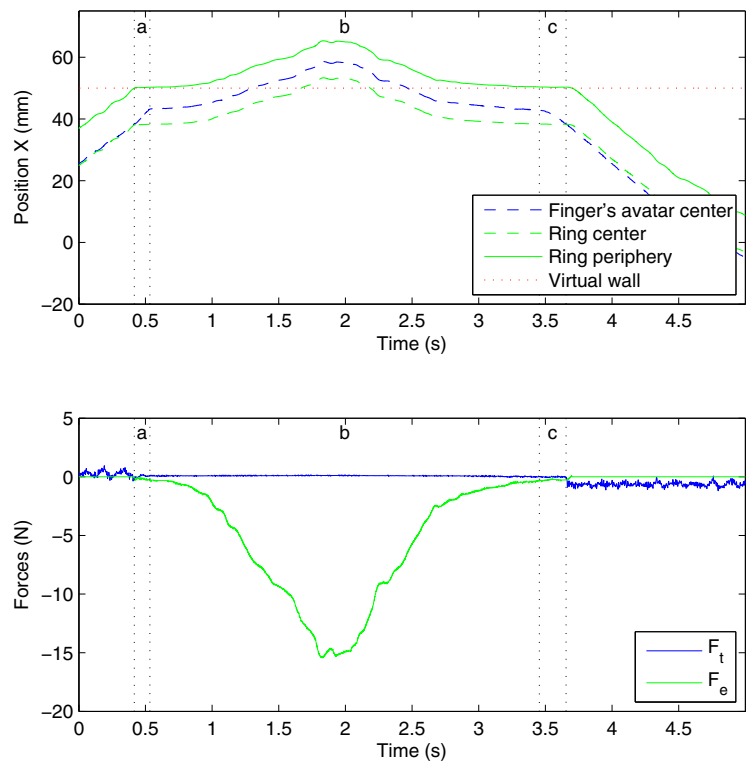

Fig. 12. 1D displacement without switch.

free space motion, and a force display mode used to render the virtual environment objects. The transition between the two modes is first managed by a hard switch triggered by the occurrence of a contact between the finger's avatar and the virtual environment, as proposed in the literature.

It has been shown that in this case the system suffers unwanted behaviors when switching between the two control modes in case of a lack of a precise on-line estimation of the finger radius. On the one hand, if the finger radius is over-estimated, some impact forces and oscillations appear during each transition between contact and free motion. On the other hand, if the finger radius is under-estimated, the operator senses a parasitic stiffness when his finger comes close to or leaves the virtual object.

A smooth transition-based control law is proposed to cope with the raised issues. The developed approach is based upon two main characteristics. First, it does not depend on the finger radius estimation. Instead, the rendered interaction force is computed based upon the ring geometry, which is constant and well known. Second, rather than switching between position tracking and force display, an adaptive gain is introduced. It moderates the influence of the tracking component in a linear way with respect to the proximity of the finger's avatar to the virtual object. An experimental comparison between both strategies shows that the proposed control law exhibits better performances while being more robust as it is independent from the finger's geometric parameters.

Future work will be twofold. First, the control law analysis will be deepened. Although no instability has been observed during the carried experiments, stability criteria will be further investigated. Second, the design of a 3D encounter-type interface will be considered, with the associated development of a whole virtual reality setup.

\section{ACKNOWLEDGMENT}

The authors wish to thank Professors Vincent Hayward and Guillaume Morel (Institut des Systèmes Intelligents et de Robotique, Sorbonne Universités, UPMC Univ Paris 06) for their valuable comments and advice during this research.

\section{REFERENCES}

[1] P. Fuchs, G. Moreau, and P. Guitton, Virtual Reality: Concepts and Technologies. CRC Press, 2011.

[2] T. Koyama, I. Yamano, K. Takemura, and T. Maeno, "Multi-fingered exoskeleton haptic device using passive force feedback for dexterous teleoperation," in Proc. IEEE/RSJ Int. Conf. on Intelligent Robots and Systems, pp. 2905-2910, 2002.

[3] M. Bouzit, G. Burdea, G. Popescu, and R. Boian, "The Rutgers Master 2 - new design force-feedback glove," IEEE/ASME Trans. Mechatronics, vol. 7, no. 2, pp. 256-263, 2002.

[4] M. Monroy, M. Oyarzabal, M. Ferre, A. Camposv, and J. Barrio, "Masterfinger: Multi-finger haptic interface for collaborative environments," in Proc. EuroHaptics, pp. 411-419, 2008.

[5] T. Endo, H. Aoyama, S. Nakagawa, and H. Kawasaki, "Haptic display by a side-faced-type multi-fingered haptic interface," in Preprint of 10th IFAC Symposium on Robot Control, pp. 367-372, 2012.

[6] T. Massie and J. Salisbury, "The PHANTOM haptic interface: A device for probing virtual objects," in Proc. ASME Symp. on Haptic Interfaces for Virtual Environment and Teleoperator Systems, pp. 295-299, 1994.

[7] D. Weir and J. Colgate, "Stability of haptic displays," in Haptic Rendering: Foundations, Algorithms and Applications, pp. 123-156, 2008.

[8] W. McNeely, "Robotic graphics: a new approach to force feedback for virtual reality," in Proc. IEEE Int'l Symp. on Virtual Reality, pp. 336 $-341,1993$.

[9] S. Tachi, T. Maeda, R. Hirata, and H. Hoshino, "A construction method of virtual haptic space," in Proc. Int'l. Conf. on Artificial Reality and Tele-Existence, pp. 131-138, 1994.

[10] Y. Yokokohji, R. L. Hollis, and T. Kanade, "What you can see is what you can feel - development of a visual/haptic interface to virtual environment," in Proc. IEEE Virtual Reality Annual Int'l Symp., pp. 46-53, 1996.

[11] T. Yoshikawa and A. Nagura, "A touch and force display system for haptic interface," in Proc. IEEE Int'l Conf. Rob. Autom., pp. 30183024, 1997.

[12] S. Nakagawara, H. Kajimoto, N. Kawakami, S. Tachi, and I. Kawabuchi, "An encounter-type multi-fingered master hand using circuitous joints," in Proc. IEEE Int'l Conf. Rob. Autom., pp. 26672672, 2005.

[13] F. Honggen, X. Zongwu, and L. Hong, "An exoskeleton master hand for controlling DLR/HIT hand," in Proc. IEEE Int'l Conf. on Intelligent Robots and Systems, pp. 3703-3708, 2009.

[14] T. Yoshikawa and A. Nagura, "A three-dimensional touch/force display system for haptic interface," in Proc. IEEE Int'l Conf. Rob. Autom., pp. 2943-2951, 1999.

[15] F. Plumet, G. Morel, and P. Bidaud, "Shall we use a dynamic model to control the motions of industrial manipulators?," in Proc. 9th World Congress on the Theory of Machines and Mechanisms, pp. 235-240, 1995.

[16] E. Larsen, S. Gottschalk, M. Lin, and D. Manocha, "Fast proximity queries with swept sphere volumes," in Proc. IEEE Int'l Conf. Robot. Autom., pp. 3719-3726, 2000.

[17] C. Ericson, Real-Time Collision Detection. CRC Press, 2004.

[18] F. Gosselin, C. Bidard, and J. Brisset, "Design of a high fidelity haptic device for telesurgery," in IEEE Interational Conference in Robotics and Automation, pp. 205-210, 2005.

[19] P. Gicquel, C. Andriot, F. Coulon-Lauture, Y. Measson, and P. Desbats, "TAO 2000 : A generic control architecture for advanced computer aided teleoperation systems," in Proc. ANS 9th Topical meeting on Robotics and remote systems, 2001.

[20] T. Greiner, "Hand anthropometry of U.S. army personnel," Tech. Rep. AD-A244 533, 1991 